\title{
Impacto na qualidade de vida em pacientes com hanseníase: correlação do Dermatology Life Quality Index com diversas variáveis relacionadas à doença
}

\section{Impact on the quality of life of patients with Hansen's disease: correlation between Dermatology Life Quality Index and disease status}

\author{
Bruna Dacier Lobato Martins ${ }^{1} \quad$ Fernanda Nogueira Torres $^{2} \quad$ Maria Leide Wand-Del-Rey de Oliveira ${ }^{3}$
}

Resumo: FundanENTOS - A hanseníase é doença infectocontagiosa crônica causada pelo Micobacterium leprae. Caracteriza-se por acometimento dermatoneurológico, variando em espectro entre dois pólos estáveis. Apesar de curável, ainda representa relevante problema de saúde pública, sendo o Brasil o segundo país mais endêmico do mundo. Sua maior morbidade associa-se aos estados reacionais e ao acometimento neural, podendo causar incapacidades físicas permanentes que comprometem significativamente a qualidade de vida dos pacientes, com auto-estigmatização e vergonha.

OBJETrVo - Avaliar o grau de comprometimento da qualidade de vida nos pacientes com hanseníase.

MÉToDos - Estudo observacional com correlação entre variáveis clínicas de gravidade da doença e questionário de avaliação da qualidade de vida: Dermatology Life Quality Index.

REsutTaDOs - Dos 40 pacientes avaliados, a maioria apresentou comprometimento da qualidade de vida de grave a muito grave segundo score obtido no Dermatology Life Quality Index. As variáveis analisadas individualmente também mostraram correlação de gravidade com o número de pontos obtido.

ConcLusão - A hanseníase causa sofrimento que ultrapassa a dor e o mal-estar estritamente vinculados ao prejuízo físico, com grande impacto social e psicológico, justificando tanto avanços para abordagem multidisciplinar ao paciente quanto a necessidade de ações de saúde que visem ao controle da doença.

Palavras-chave: Doenças endêmicas; Hanseníase; Qualidade de vida; Questionários

\begin{abstract}
BACKGROUND - Hansen's disease is a chronic infecto-contagious illness caused by Mycobacterium leprae. It is characterized by dermato-neurological damage, varying in spectrum between two steady poles. Albeit curable, it still represents an important public bealth problem, Brazil being the second most endemic country in the world. Its higher morbidity is associated with reactions and neural damage, which is able to cause permanent disabilities that significantly compromising the quality of life of the patients, with self-stigmatization and shame.

OBJECTVE - To evaluate the degree of compromise in the quality of life of patients with Hansen's disease.

MEтноDS - Observational study with correlation between clinical indicators of severity and a questionnaire on quality of life: the Dermatology Life Quality Index (DLQI).

REsLITS - Of the 40 evaluated patients, the majority presented an impairment in the quality of life, which ranged from the serious to the very serious score in the Dermatology Life Quality Index. Individual analysis of disease indicators also showed a correlation between severity and the Dermatology Life Quality Index.

ConcLusion - Hansen's disease causes suffering that exceeds the pain and the malaise strictly related to the physical damage, having a great social and psychological impact. This justifies both improvements to a multidisciplinary bealth care approach and the need of bealth actions aiming at disease control.

Keywords: Endemic disease; Hansen's disease; Quality of life; Questionnaires
\end{abstract}

\footnotetext{
Recebido em 13.11.2006.

Aprovado pelo Conselho Consultivo e aceito para publicação em 09.09.2007

* Trabalho realizado no ambulatório de Hansenologia do Hospital Universitário Clementino Fraga Filho - Universidade Federal do Rio de Janeiro (HUCFF-UFRJ)

- Rio de Janeiro (RJ), Brasil.

Conflito de interesse: Nenhum / Conflict of interest: None

Suporte financeiro: Nenhum / Financial funding: None

Pós-graduanda em dermatologia. Hospital Universitário Clementino Fraga Filho - Universidade Federal do Rio de Janeiro (HUCFF-UFRJ) - Rio de Janeiro (RJ), Brasil. Residente em dermatologia. Hospital Universitário Clementino Fraga Filho - Universidade Federal do Rio de Janeiro (HUCFF-UFRJ) - Rio de Janeiro (RJ), Brasil. Professora doutora, adjunta da faculdade de medicina. Hospital Universitário Clementino Fraga Filho -Universidade Federal do Rio de Janeiro (HUCFF-UFRJ) Rio de Janeiro (RJ), Brasil.
}

(C)2008 by Anais Brasileiros de Dermatologia 


\section{INTRODUÇÃO}

A hanseníase é doença infectocontagiosa crônica causada pelo Mycobacterium leprae. Caracteriza-se por acometimento dermatoneurológico, variando em espectro entre dois pólos estáveis (tuberculóide e virchowiano), com formas intermediárias instáveis. Uma classificação operacional, para fins de tratamento, reúne os doentes em dois grupos: a) paucibacilares (PB): correspondem a formas clínicas que possuem imunidade celular preservada, baciloscopia negativa e teste de Mitsuda positivo; b) multibacilares (MB): correspondem a formas clínicas com imunidade específica ao bacilo reduzida ou ausente e baciloscopia positiva. De acordo com essa classificação, define-se o tratamento com a poliquimioterapia (PQT) estabelecida pela Organização Mundial de Saúde (OMS) ${ }^{1,2}$ Apesar de curável, a hanseníase ainda representa relevante problema de saúde pública, sendo o Brasil o segundo país mais endêmico no mundo. ${ }^{3}$ Sua maior morbidade associa-se aos estados reacionais e ao acometimento neural que podem causar incapacidades físicas e deformidades permanentes, comprometendo significativamente a qualidade de vida dos pacientes, com auto-estigmatização e vergonha. Esse problema se agrava pelo fato de a enfermidade estar historicamente associada a estigmas, o que mantém na representação social a idéia de doença mutilante e incurável, provocando atitudes de rejeição e discriminação ao doente, com sua eventual exclusão da

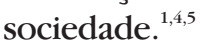

O conceito de saúde é muito amplo, abrangendo diversos aspectos da vida diária de um indivíduo. Segundo a OMS "é a percepção do indivíduo sobre seu bem-estar físico, mental e social, e não meramente a ausência de doenças". Como é conceito subjetivo, apesar de muitos médicos considerarem a importância do comprometimento da qualidade de vida de seus pacientes, essa avaliação, infelizmente, não é tão acurada em demonstrar o real impacto. Médico e paciente costumam ter diferentes avaliações sobre o estado de saúde de um mesmo paciente..$^{6.8}$ Nas últimas décadas vem crescendo o interesse médico nesse campo, com desenvolvimento de diversos questionários de avaliação da qualidade de vida relacionados à saúde visando a medidas objetivas do problema e suas conseqüências, a fim de uniformizar essas diferentes visões, permitindo comparações inter e intrapessoais, e melhor abordagem terapêutica dos pacientes. Estes se dividem em "Instrumentos Genéricos" (The medical outcomes study 36-item short form health survey: SF-36, EuroQoi 5-D: EQ-5D), "Áreaespecíficos" (Dermatology Life Quality Index: DLQI, Dermatology Quality of Life Scales: DqoLS), e "Doençaespecíficos" (Psoriasis Disability Index: PDI, Activities of Daily Vision Scale: ADVS).'

O Dermatology Life Quality Index (DLQI), primeiro questionário de avaliação da "qualidade de vida relacionada à saúde" específico à dermatologia, foi desenvolvido por Finlay e Khan em 1994, traduzido e validado para a língua portuguesa,${ }^{10,11} \mathrm{e}$ vem sendo aplicado em várias dermatoses, como psoríase, dermatite atópica, urticária, acne, entre outras,${ }^{12}$ sendo a primeira vez que se aborda esse aspecto na hanseníase.

\section{MATERIAL E MÉTODOS}

Estudo observacional, clínico-epidemiológico, transversal, em que foram avaliados ambulatorialmente 40 pacientes de ambos os sexos, com idade superior a 18 anos e diagnóstico de hanseníase clínico e/ou histopatológico, em tratamento com PQT ou pós-alta. Essa avaliação compreendeu anamnese pesquisando a presença de episódios reacionais hansênicos durante toda evolução clínica do doente; exame clínico completo para classificação em pauci (PB) ou multibacilar (MB); teste de sensibilidade; rotina de avaliação neurológica e do grau de incapacidade física (GIF). Aplicouse o DLQI a todos os pacientes, sendo esse questionário composto por 10 questões que envolvem aspectos da vida diária e resulta em scores interpretados como: sem comprometimento da qualidade de vida (0-1) ou com comprometimento leve (2-5), moderado (6-10), grave (11-20) ou muito grave (21-30). ${ }^{10,11}$ O score obtido na aplicação do DLQI foi correlacionado às diversas variáveis abordadas: forma clínica pauci ou multibacilar; presença de pelo menos um episódio reacional (tipo I ou II) e grau de incapacidade física $(0,1$ ou 2$)$. A análise estatística foi processada pelo Epinfo2000, e o critério de significância adotado foi de $5 \%(p<0,05)$.

\section{RESULTADOS}

Em meio aos 40 pacientes que participaram do estudo, encontraram-se $60 \%$ do sexo feminino e $40 \%$ do sexo masculino; com idade mínima de 19 e máxima de 74 anos (média de 40 anos). Desses, $70 \%$ foram classificados como multibacilares, e $30 \%$ paucibacilares; $65 \%$ apresentaram pelo menos um episódio reacional durante a evolução clínica da doença. A maioria dos pacientes multibacilares $(92,9 \%)$ apresentou reação hansênica em algum momento de sua evolução. Em relação ao grau de incapacidade física (GIF), 36,8\% corresponderam a $0,44,7 \%$ a 1 e $18,4 \%$ a 2 . Oitenta e cinco por cento dos pacientes com algum grau de incapacidade física (GIF 1 ou 2) apresentaram episódio reacional no decorrer de sua evolução clínica. Os scores obtidos na aplicação do DLQI variaram de 1 a 25 pontos (média 11 pontos), e a maioria dos pacientes (45\%) apresentou score na dimensão do grave ou muito grave; $40 \%$ dos pacientes do leve ao moderado, e apenas $15 \%$ dos pacientes não informaram comprometimento da qualidade de vida (Gráfico 1). Dos pacientes paucibacilares, $50 \%$ não informaram comprometimento da 
Gráfico 1: Distribuição da amostra segundo classificação pelo Dermatology life quality index

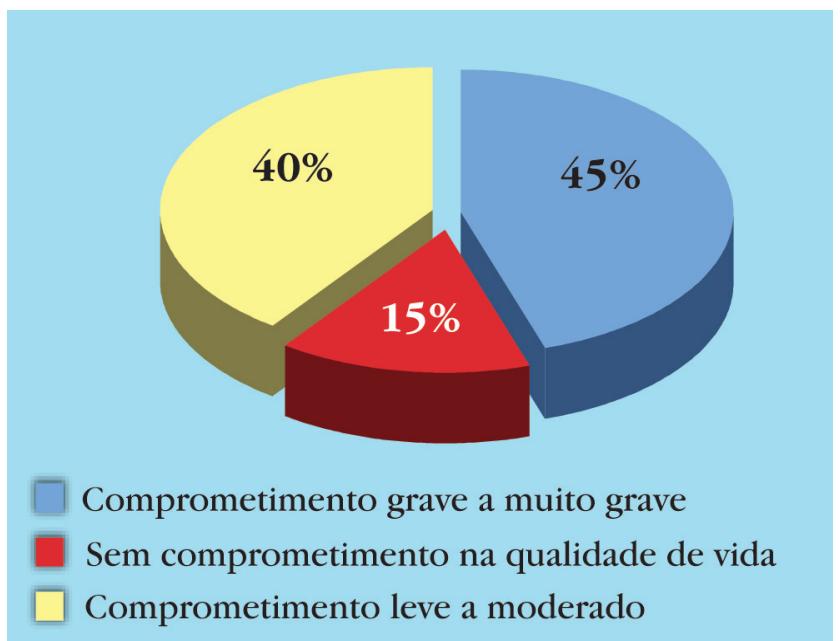

GRÁFICo 2: Correlação Dermatology life quality index e formas clínicas

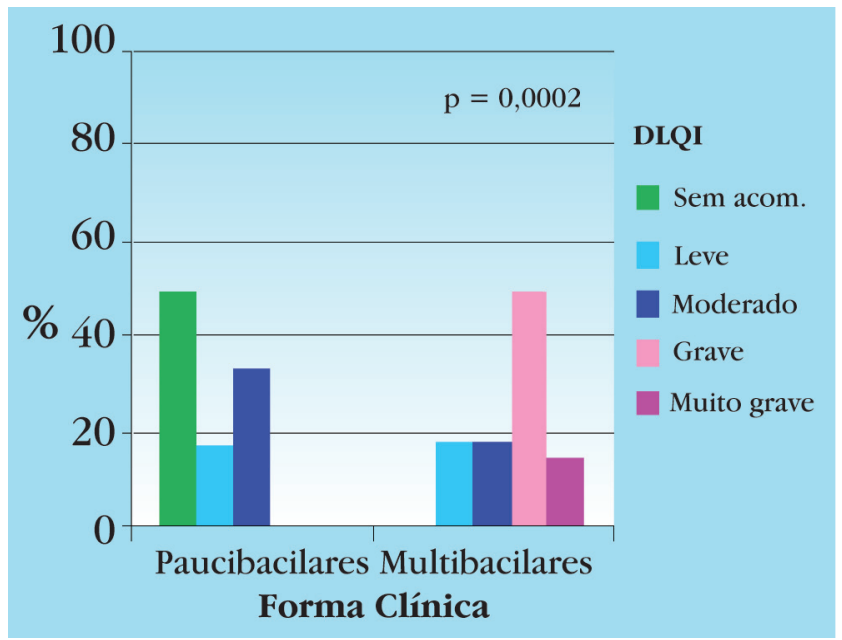

qualidade de vida, e os demais apresentaram apenas acometimento leve a moderado. Já em relação aos multibacilares, $100 \%$ dos pacientes apresentaram algum grau de prejuízo da qualidade de vida, e a maioria deles (65\%) obteve score na condição grave ou muito grave ao DLQI, $\mathrm{p}=0,0002$ (Gráfico 2). Quanto ao grau de incapacidade física, mais de $50 \%$ dos pacientes acometidos (GIF 1 ou 2) apresentaram score grave ou muito grave, contrastando com os pacientes que não possuem incapacidade física, $\mathrm{p}=0,254$ (Gráfico 3). A presença de reação hansênica correlacionou-se em todos os casos com algum grau de comprometimento da qualidade de vida, tendo $65 \%$ desses pacientes score na dimensão do grave ou muito grave ao DLQI. Já os sem história de reação apresentaram-se em sua maioria sem prejuízo da qualidade de vida, $\mathrm{p}=0,0009$ (Gráfico 4).
GráfICo 3: Correlação Dermatology life quality index e grau de incapacidade física

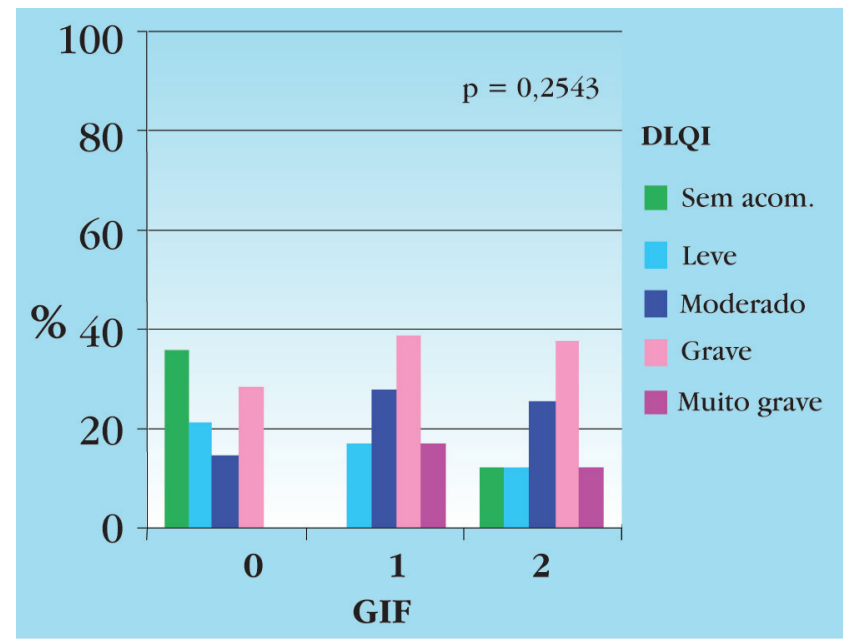

GráFICO 4: Correlação Dermatology life quality index e reação hansênica

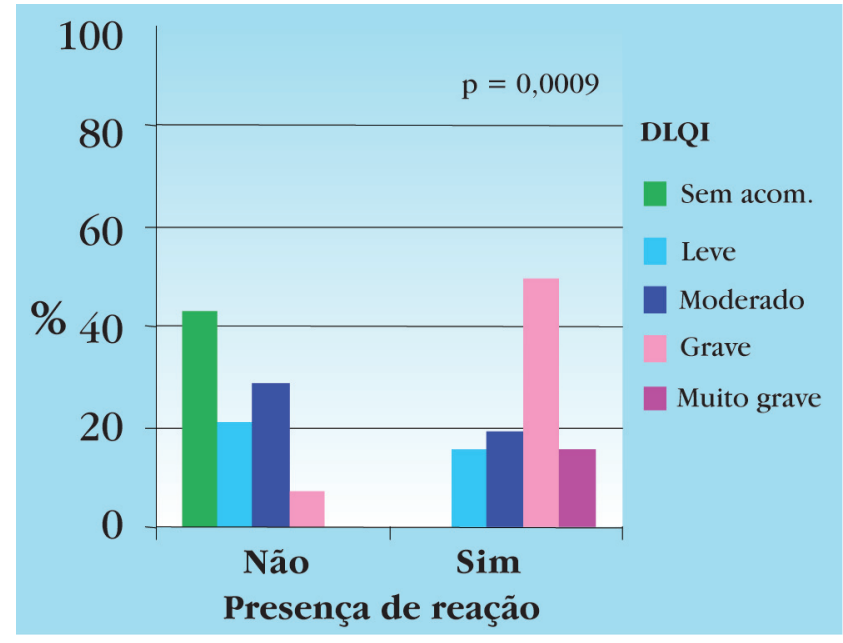

Quando a amostra é agrupada em pacientes com ou sem incapacidade física e correlacionada ao Dermatology life quality index, atinge significância estastística $(\mathrm{p}<0,05)$. Para efeito de ilustração, o grupo foi dividido segundo grau de incapacidade: GIF 0,1 ou 2. GIF = grau de incapacidade física DLQI=dermatology life quality index

\section{DISCUSSÃO}

A partir da interpretação dos dados deste estudo, reitera-se o importante impacto da hanseníase na qualidade de vida de seus portadores, tal como indicado pelos altos scores obtidos na aplicação do DLQI (média de 11 pontos), comparáveis aos de doenças crônicas, como a psoríase, dermatite atópica e acne. Esse questionário de qualidade de vida vem sendo amplamente utilizado como parâmetro de gravidade em diversas dermatoses, psoríase e dermatite atópica ocupando lugar 
TABela 1: Comparação de scores nos diversos diagnósticos em estudos de validação do Dermatology life quality index

\begin{tabular}{|c|c|c|c|c|c|}
\hline Diagnósticos & $\begin{array}{l}\text { DLQI-BRA } \\
(\mathrm{n}=241) \\
\text { média } \\
\text { (desvio padrão) }\end{array}$ & $\begin{array}{l}\text { DLQI-USA } \\
(\mathbf{n}=200) \\
\text { intervalo }\end{array}$ & $\begin{array}{l}\text { DLQI-DIN } \\
(\mathbf{n}=200) \\
\text { média }\end{array}$ & $\begin{array}{l}\text { DLQI-ESP } \\
(\mathrm{n}=237) \\
\text { intervalo }\end{array}$ & $\begin{array}{l}\text { DLQI-UK }(n=200) \\
\text { média (desvio padrão) } \\
\text { intervalo }\end{array}$ \\
\hline Psoríase & $10,31(6,96)$ & $2-30$ & 4,6 & $1-14$ & $\begin{array}{ll}13,3 & (8,7) \\
2-30 & \end{array}$ \\
\hline Acne & $7,40 \quad(5,00)$ & $1-27$ & 9 & $0-19$ & $\begin{array}{l}6,1 \\
1-13\end{array}$ \\
\hline Dermatite atópica & $7,87 \quad(5,11)$ & $0-16$ & 5,8 & $1-9$ & $\begin{array}{l}16,2 \\
2-26\end{array} \quad(7,4)$ \\
\hline
\end{tabular}

Fonte: Zogbi $\mathrm{H}$ et al. ${ }^{13}$

de destaque em função do grande prejuízo que provocam na vida diária de seus pacientes. ${ }^{13} \mathrm{Na}$ avaliação de pacientes com psoríase, ao lado de outros parâmetros de gravidade específicos da doença, impõe-se a regra dos 10, isto é, quando o score obtido no DLQI é maior do que 10 pontos, o quadro é classificado como grave. ${ }^{14}$ A média dos valores obtidos no DLQI em diferentes doenças varia conforme a amostra estudada; em estudo brasileiro de Zogbi \& Muller, a média para pacientes com psoríase foi 10,31 , para aqueles com dermatite atópica, 7,87, e para aqueles com acne, 7,40, asseme lhando-se aos valores encontrados no estudo original de Finlay \& Khan (Tabela 1).

Os resultados também apontam forte relação do maior comprometimento na qualidade de vida dos portadores de hanseníase com a forma clínica multibacilar, presença de algum grau de incapacidade física e reação hansênica, como observado nos gráficos 2,3 e 4, respectivamente. As correlações obtidas entre as variáveis propostas atingiram significância estatística, e em relação à presença de incapacidade física, ao se agruparem os pacientes com graus 1 e 2 de incapacidade em um mesmo grupo, observou-se significância estatística na correlação com o score obtido no DLQI $(p<0,05)$. Esses achados refletiram de forma real e objetiva a avaliação subjetiva do médico sobre o comprometimento da qualidade de vida nesta amostra, que se mostrou reproduzível ao longo do tempo em quadros com progressão estável, sendo diretamente variável com a melhora ou piora do quadro. Devido aos aspectos clínicos dessa enfermidade - variedade de apresentação, presença de reações hansênicas e caráter incapacitante -, ao grave comprometimento na qualidade de vida e à visão histórica estigmatizante, evidencia-se a importância de se avaliar qualitativamente o impacto na qualidade de vida dos pacientes, objetivando visão global e permitindo avaliação mais profunda do que a resultante apenas da análise clínica, com conseqüentes avanços na abordagem da pessoa atingida pela hanseníase.

\section{CONCLUSÃO}

A hanseníase causa grande prejuízo para a vida diária e as relações interpessoais, provocando sofrimento que ultrapassa a dor e o mal-estar estritamente vinculados ao prejuízo físico, com grande impacto social e psicológico, como demonstrado pela aplicação do questionário de qualidade de vida proposto (DLQI). Esse prejuízo na qualidade de vida associou-se de forma mais importante a algumas situações específicas da doença, como forma clínica multibacilar, reação hansênica e incapacidade física. Fazem-se, portanto, necessários abordagem multidisciplinar ao paciente, ações que visem não só à eliminação, mas também à prevenção de incapacidades, estímulo à adesão ao tratamento e combate ao estigma social, a fim de minimizar o impacto da doença sobre a vida do indivíduo. ${ }^{1,2,4,5}$ 


\section{REFERÊNCIAS}

1. Talhari S, Neves RG, Penna GO, de Oliveira MLV. Hanseníase. 4 ed. Manaus: Gráfica Tropical; 2006. 215 p.

2. Brasil. Ministério da Saúde. Secretaria de políticas de saúde. Departamento de atenção básica. Guia para o controle da hanseníase. Brasília: Ministério da Saúde; 2002. 89p.

3. Rao PN. Recent advances in the control programs and therapy of leprosy. Indian J Dermatol Venereol Leprol. 2004;70:269-76.

4. Singhi MK, Ghiya BC, Gupta D, Kachhawa D. Disability rates in leprosy. Indian J Dermatol Venereol Leprol. 2004;70:314-16.

5. Kaufmann A, Mariam SG, Neville J. A dimensão social da hanseníase: manual prático para trabalhadores da saúde. Itália: Associazione Italiana Amici di Raoul Follereau; 1982. 110p.

6. Hallioua B, Beumont MG. Quality of life in dermatology. Int J Dermatol. 2000;39:801-6.

7. Hornquist JO. The concept of quality of life. Scand J Soc Med. 1982;10:57-61.

8. Finlay AY, Ryan Tj. Disability and handicap in dermatology. Int J Dermatol. 1996;35:305-11.

9. Finlay AY. Quality of life indices: review article. Indian J Dermatol Venereol Leprol. 2004;70:143-8.

10. Ukdermatology.co [homepage on the Internet]. Cardiff: Departament of Dermatology, Wales collegue of medicine, Cardiff University; c2004-06 [updated] 2007 Jan]. Available from: http://www.ukdermatology.co.uk/.

11. Finlay AY, Khan GK. Dermatology life quality index (DLQI)-a simple practical measure for routine clinical use. Clin Exp Dermatol. 1994;19:210-6.

12. Lewis V, Finlay AY. Ten years experience of dermatol ogy life quality index (DLQI). J Investig Dermatol Symp Proc. 2004;9:169-80.

13. Zogbi H, Muller MC, Protas JS, Kieling CM, Driemeier FM. Adaptação e validação DLQI (Dermatology Life Quality Index) para uma amostra brasileira: avaliando qualidade de vida em dermatologia. PsiqWeb [periódico on-line]. [acesso 24 Jan 2008]. Disponível em: http://www.virtualpsy.org/psicossomat ica/dermato3.html.

14. Finlay AY. Current severe psoriasis and the rule of tens. Br J Dermatol. 2005;152:861-67.

ENDEREÇO PARA CORRESPONDÊNCIA / MAILING ADDRESS:

Bruna Dacier Lobato Martins

Estrada da Gávea 847/1003 - São Conrado

22610-970 - Rio de Janeiro - RJ

Tel./Fax: (21) $33226511 / 91519844$

E-mail: brunadlm@botmail.com

Como citar este artigo / How to cite this article: Martins BDL, Torres FN, Oliveira MLW. Impacto na qualidade de vida em pacientes com hanseníase: correlação do Dermatology Life Quality Index com diversas variáveis relacionadas à doença. An Bras Dermatol. 2008;83(1):39-43 